\title{
Impact of food processing on total polyphenol content and in vitro antioxidant activity in different sorghum varieties
}

\author{
C. Bösch ${ }^{1}$, N. Watson ${ }^{2}$ and C. Orfila ${ }^{1}$ \\ ${ }^{1}$ School of Food Science and Nutrition, University of Leeds, Leeds, LS2 9JT, UK and ${ }^{2}$ Department of Chemical and \\ Environmental Engineering, University of Nottingham, Nottingham, NG2 2RD, UK
}

Sorghum is one of the most widely cultivated crops worldwide, and, due to its adaptation to low input agricultural systems, is of particular importance as a staple food for many sub-Saharan countries. It provides a valuable source of starch, protein, minerals and polyphenols to local diets. However, sorghum requires significant water and labor-intensive pre-processing methods to increase the otherwise limited bioavailability of nutrients for human consumption ${ }^{1,2}$. The aim of this project was to compare the effect of different processing conditions on the total polyphenol content and associated bioactivity levels in a range of differently pigmented Sorghum cultivars sourced from two sub-Saharan regions.

Purified grain samples were milled and subjected to traditional fermentation, microwaving or boiling in order to produce sorghum porridge. After extraction with a mixture of $1 \% \mathrm{HCl}$ in methanol, the total polyphenol content was determined using the Folin Ciocalteu assay; two antioxidant assays, TEAC and DPPH, were performed using standard protocols.

Overall, the total polyphenol content (Figure 1), as well as antioxidant activity (data not shown) correlated well and were largely dependent on the country of origin and the grain pigmentation, being significantly higher in red pigmented varieties as well as in cultivars sourced from Uganda (Serena, Seredo, Seso 1, Lulu-1). Bioactive levels in fermented porridge samples were markedly higher compared to boiled and microwaved samples and only slightly lower than in unprocessed flour.

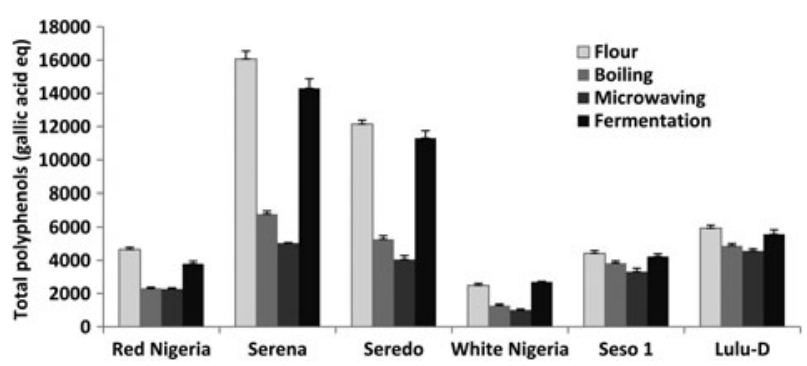

Figure 1: Effect of different food processing methods on total polyphenol content in selected Sorghum samples from sub-Saharan origin.

In summary, when comparing grains grown within the same country, high pigmentation levels correspond with high levels of polyphenols. Furthermore, traditional fermentation procedures were more efficient than boiling and microwaving techniques in retaining polyphenol content. Therefore, traditional fermentation methods have the potential to provide higher levels of polyphenols which are associated with health promoting effects.

1. De Morais Cardoso L, Aguiar-Monini T, Silva Pinheiro S et al. (2014) J Food Chem 152, 210-217

2. Wang T, He F \& Chen G (2014) J Functional Foods 7, 101-111 